\title{
A THEORY OF SECANT PRECONDITIONERS
}

\author{
JOSÉ MARIO MARTÍNEZ
}

\begin{abstract}
In this paper we analyze the use of structured quasi-Newton formulae as preconditioners of iterative linear methods when the inexact-Newton approach is employed for solving nonlinear systems of equations. We prove that superlinear convergence and bounded work per iteration is obtained if the preconditioners satisfy a Dennis-Moré condition. We develop a theory of LeastChange Secant Update preconditioners and we present an application concerning a structured BFGS preconditioner.
\end{abstract}

\section{INTRODUCTION}

Newton's method is the best-known algorithm for solving nonlinear systems of equations

$$
F(x)=0,
$$

where $F: \mathbb{R}^{n} \rightarrow \mathbb{R}^{n}$ is differentiable (see $\left.[11,32,33,39]\right)$. We denote $J(x) \equiv$ $F^{\prime}(x)$. At each iteration of this method, the linear system

$$
J\left(x_{k}\right) s_{k}=-F\left(x_{k}\right)
$$

is solved, and the new approximation to the solution of $(1.1)$ is defined by

$$
x_{k+1}=x_{k}+s_{k} \text {. }
$$

Under suitable assumptions, Newton's method is locally and quadratically convergent to isolated solutions of (1.1). Because of this property, the Newton method is the most suitable algorithm for many practical problems.

The linear system (1.2) is usually solved employing $L U$ or $Q R$ factorizations (see $[16,19$, etc.]). When $n$ is large and $J(x)$ is sparse, $L U$ techniques are preferred (see $[17,20,15,45$, etc.]), However, for many sparsity patterns that appear frequently in applications (e.g., in the discretization of 3-D boundary value problems) the $L U$ factorization and its variations produce an unacceptable amount of fill-in. Therefore, both the computer time and memory requirements that are necessary to solve (1.2) turn out to be very large. In these cases it is generally preferred to use an iterative method in order to obtain an approximation of the solution of (1.2). The advantage of iterative linear methods is that the storage required to implement them is essentially the same as

Received by the editor July 11, 1991 and, in revised form, March 3, 1992.

1991 Mathematics Subject Classification. Primary 65H10.

Key words and phrases. Nonlinear systems, inexact-Newton methods, quasi-Newton methods, preconditioners.

Work supported by FAPESP (Grant 90/3724-6), FINEP, CNPq and FAEP (UNICAMP). 
that required to store the data of the problem. Moreover, the computer time consumed by a single iteration of most linear iterative methods is negligible compared with the computer time used by direct methods. Often, the application to (1.2) of a moderate number of steps of a linear iterative method is sufficient to provide satisfactory progress towards the solution of the nonlinear system (1.1). For instance, if $x_{k}$ is not close to a solution of (1.1), it is hardly worthwhile to waste a lot of computer time solving accurately (1.2), since we do not expect much improvement in the approximation from an accurate solution of (1.2). See [35] and [34] for a theoretical analysis of the behavior of Newton's method far away from the solution.

Many authors (see [32, 40] and the references in [6]) analyzed the behavior of methods based on the application to (1.2) of a predetermined number of iterations of some linear iterative method. Ortega and Rheinboldt call algorithms based on this idea "Generalized Linear Methods". However, only in 1982, Dembo, Eisenstat, and Steihaug [6] gave a satisfactory answer to the question of deciding when the number of linear iterations executed at the $k$ th step of the nonlinear method is sufficient. The algorithms based on their idea are called "inexact-Newton methods".

The Dembo-Eisenstat-Steihaug criterion consists of defining $s_{k}$ as any increment that satisfies

$$
\left|J\left(x_{k}\right) s_{k}+F\left(x_{k}\right)\right| \leq \theta_{k}\left|F\left(x_{k}\right)\right|,
$$

where $0<\theta_{k}<\theta<1$ and $|\cdot|$ is some norm on $\mathbb{R}^{n}$. Under suitable conditions, Dembo, Eisenstat, and Steihaug proved that the method defined by (1.4) and (1.3) has local linear convergence in an appropriate norm, and that convergence is superlinear if $\lim _{k \rightarrow \infty} \theta_{k}=0$.

The theory of Dembo, Eisenstat, and Steihaug is useful to analyze cases where the equation (1.2) is solved inaccurately for different reasons (see [5]). In this work we are concerned with the case where the reason for inaccuracy is the use of an iterative linear method.

In the last 15 years the most widely used iterative methods for solving linear systems have been the Conjugate Direction methods (see [21, 18, 19, 44, 13] and references therein). Satisfactory practical behavior of these methods for solving a general linear system $A s=b$ depends, in most cases, on the judicious choice of a preconditioning procedure. Roughly speaking, a preconditioning technique consists of finding an equivalent linear system $A^{\prime} s=b^{\prime}$ such that the new system is easier to solve than the original one by the iterative linear method and the transformation of $A s=b$ onto $A^{\prime} s=b^{\prime}$ is computationally cheap. Thus, the idea of most preconditioning methods is to find "cheap approximations" to the inverse or to the $L U$ factorization of the matrix (see [19, $\S 10.3],[1])$. Frequently, the preconditioning matrix is an incomplete sparse $L U$ factorization of $A$, or is produced by a fixed number of applications of some convergent stationary linear iterative method.

Let us call $B_{k}^{-1}$ the approximation of $J\left(x_{k}\right)^{-1}$ used at each iteration of an inexact-Newton method for preconditioning the linear system (1.2). We define the following "inexact-Newton method with explicit preconditioning":

Algorithm 1.1. Given $x_{0} \in \mathbb{R}^{n}, B_{0} \in \mathbb{R}^{n \times n}, \theta_{k} \in(0,1), k=0,1,2, \ldots$, the steps of a typical iteration of this algorithm are the following: 
Step 1. Apply a linear iterative method to the system (1.2), using $B_{k}$ as preconditioner. Stop when (1.4) is satisfied.

Step 2. Define

$$
x_{k+1}=x_{k}+s_{k} .
$$

Step 3. Using $B_{k}, J\left(x_{k+1}\right), F\left(x_{k+1}\right), F\left(x_{k}\right), s_{k}$ and perhaps additional information available, compute a new preconditioner $B_{k+1}$.

The key point of Algorithm 1.1 is at Step 3. At this step we compute the preconditioner for the new linear system. We observe that information at the previous iteration is available and so there is no reason for not using it. In other words, the linear systems that must be solved at each iteration of the inexactNewton method are not isolated, and hence useful information can be passed between iterations in order to improve the quality of the preconditioner. An efficient way to use the previous available information is to use preconditioners $B_{k}$ that satisfy the "secant equation" (see $[3,9,10,11,14,26$, etc.])

$$
B_{k+1} s_{k}=y_{k} \text {, }
$$

where

$$
y_{k}=F\left(x_{k+1}\right)-F\left(x_{k}\right) .
$$

Nazareth and Nocedal [31] and Nash [29, 30] were the first in using preconditioners based on (1.5) in connection with unconstrained minimization problems.

If (1.5) is satisfied, global information on the true Jacobians is incorporated in the new preconditioner, by virtue of the identity

$$
y_{k}=\left(\int_{0}^{1} J\left(x_{k}+t s_{k}\right) d t\right) s_{k} .
$$

This contrasts with the usual approach of preconditioning using only the current Jacobian, that, typically, uses only partial information about $J$ (diagonal preconditioners and incomplete $L U$ preconditioners are typical examples of this case. See [4]).

A combined approach is to use classical (incomplete) preconditioners associated with least-change secant updates $([11,12,14,25])$.

The most natural "combined preconditioners" are "structured least-change secant update" matrices in the sense of [14] and [26]. In this case,

$$
B_{k}=C\left(x_{k}\right)+A_{k},
$$

where $C\left(x_{k}\right)$ includes partial information on $J\left(x_{k}\right)$, and $A_{k}$ is updated using least-change secant update techniques $[11,14]$. Of course, for $(1.7)$ to be useful as preconditioner, the inversion of $B_{k}$ must be inexpensive. This can be achieved, for example, if $C\left(x_{k}\right)=L_{k} U_{k}$, where $L_{k} U_{k}$ is an incomplete $L U$ factorization of $J\left(x_{k}\right)$, and $A_{k}$ is a low-rank matrix.

Practical experience showed that some secant update procedures generate useful preconditioners for the Newton equation (1.2) (see [29, 30]). The existence of powerful convergence theories of secant methods for nonlinear systems suggests that a comprehensive theory of secant preconditioners can also be developed. Some steps in that direction were made by Martinez [26]. He defined a general algorithm in which $x_{k+1}$ is any point that satisfies

$$
\left|x_{k+1}-x_{k}^{N}\right| \leq\left|x_{k}^{Q}-x_{k}^{N}\right|
$$


where

$$
x_{k}^{N}=x_{k}-J\left(x_{k}\right)^{-1} F\left(x_{k}\right)
$$

and $x_{k}^{Q}$ is obtained using a least-change secant update (LCSU) procedure. Then, he proved that under the same hypotheses that guarantee the local convergence of the "pure" LCSU method, the local convergence of the process based on (1.8) may also be proved. When the classical Conjugate Gradient Method is applied to the problem

$$
\underset{s}{\operatorname{Minimize}}\left\|B_{k}^{-1}\left[J\left(x_{k}\right) s+F\left(x_{k}\right)\right]\right\|_{2}
$$

starting with $s_{k}^{0}=x_{k}^{Q}-x_{k}$, and generating a sequence $\left\{s_{k}^{1}, s_{k}^{2}, \ldots\right\}$, it is well known (see [21]) that

$$
\left\|s_{k}^{j+1}-s_{k}^{N}\right\|_{2} \leq\left\|s_{k}^{j}-s_{k}^{N}\right\|_{2}
$$

for all $j=0,1,2, \ldots$, where $s_{k}^{N}$ is the exact solution of (1.9). By (1.10), the property (1.8) holds for $|\cdot|=\|\cdot\|_{2}$ if $s_{k} \in\left\{s_{k}^{0}, s_{k}^{1}, s_{k}^{2}, \ldots\right\}$.

The approach of Martinez [26] has two main drawbacks. First, the condition (1.8) restricts the choice of the iterative linear method to algorithms where the "norm-decreasing property" (1.10) holds. Unhappily, this property is not true for many successful iterative methods such as GMRES ([36, 37]), and it also fails to hold if the preconditioning procedure of the classical CG algorithm involves a change of variables, as it usually does (see Algorithm 10.3.1 in [19]). Second, the conditions for local convergence of LCSU methods require that the first preconditioner $B_{0}$ must be close to the Jacobian. If we are using an incomplete $L U$, or a preconditioner based on a stationary linear iterative method, this condition can be very restrictive. These difficulties led us to ask for the possibility of defining LCSU-preconditioned inexact-Newton methods where superlinear convergence is obtained using a bounded number of steps of an unspecified linear iterative method at each iteration, and where the assumption of a good initial $B_{0}$ is not necessary for proving local convergence. In $\S 2$ of this paper we prove that we are able to define an algorithm with these characteristics if the preconditioners satisfy the Dennis-Moré condition (see [9]), and $\left|B_{k}\right|,\left|B_{k}^{-1}\right|$ are bounded. In $\S 3$ we prove that preconditioners which obey Martinez's theory with null ideal parameter $r_{*}$ generate algorithms that satisfy the conditions given in $\S 2$. So, structured least-change secant update methods in the sense of Dennis and Walker can also be used for that purpose (see [27]). In $\S 4$ we apply the theory of $\S 3$ to a structured BFGS preconditioner. Some conclusions are given in $\S 5$.

\section{Preconditioning With the Dennis-Moré condition}

When we want to solve a linear system $A s=b$ using an iterative linear method with the preconditioner $B^{-1} \approx A^{-1}$, it is natural to begin testing $\tilde{s}=$ $B^{-1} b$. This trial point is incorporated in a natural way in the description of preconditioned $C G$ algorithms (see, for example, Algorithm 10.3.1 in [19]). The algorithm that we present below incorporates explicitly that trial point in the inexact-Newton context.

From now on, $|\cdot|$ denotes a norm on $\mathbb{R}^{n}$ and its subordinate matrix norm. 
Algorithm 2.1. Let $\theta_{k} \in(0, \theta)$ for all $k=0,1,2, \ldots, 0<\theta<t<1$, and $\lim _{k \rightarrow \infty} \theta_{k}=0$. Assume that $x_{0} \in \mathbb{R}^{n}$ is an initial approximation to the solution of (1.1) and $B_{0} \in \mathbb{R}^{n \times n}$ is an initial preconditioner. Given $x_{k} \in \mathbb{R}^{n}$ and $B_{k} \in \mathbb{R}^{n \times n}$, the steps for obtaining $x_{k+1}, B_{k+1}, k=0,1,2, \ldots$, are the following:

Step 1. If $B_{k}$ is nonsingular, compute

$$
s_{k}^{Q}=-B_{k}^{-1} F\left(x_{k}\right) \text {. }
$$

Else, go to Step 3.

Step 2. If

$$
\left|J\left(x_{k}\right) s_{k}^{Q}+F\left(x_{k}\right)\right| \leq \theta\left|F\left(x_{k}\right)\right|,
$$

define

$$
s_{k}=s_{k}^{Q}
$$

and go to Step 4.

Step 3. Find an increment $s_{k}$ such that

$$
\left|J\left(x_{k}\right) s_{k}+F\left(x_{k}\right)\right| \leq \theta_{k}\left|F\left(x_{k}\right)\right|,
$$

using some iterative method.

Step 4. Define

$$
x_{k+1}=x_{k}+s_{k}
$$

and compute a new preconditioner $B_{k+1}$.

Clearly, Algorithm 2.1 is a particular case of the inexact-Newton method of Dembo, Eisenstat, and Steihaug. So, it is linearly convergent in the norm defined by $|z|_{*}=\left|J\left(x_{*}\right) z\right|$, under appropriate conditions on $F$ and $x_{0}$. Let us state these assumptions precisely.

Assumption 1. Assume that $F: \Omega \subset \mathbb{R}^{n} \rightarrow \mathbb{R}^{n}, \Omega$ an open and convex set, $F \in C^{1}(\Omega), x_{*} \in \Omega, J\left(x_{*}\right)$ nonsingular and $F\left(x_{*}\right)=0$. Assume that there exists $L \geq 0$ such that, for all $x \in \Omega$,

$$
\left|J(x)-J\left(x_{*}\right)\right| \leq L\left|x-x_{*}\right|
$$

Inequality (2.6) implies that for all $x, z \in \Omega$,

$$
\left|F(z)-F(x)-J\left(x_{*}\right)(z-x)\right| \leq L|z-x| \sigma(x, z),
$$

where

$$
\sigma(x, z)=\max \left\{\left|x-x_{*}\right|,\left|z-x_{*}\right|\right\}
$$

(See [3].)

The following theorem is a trivial consequence of the Theorem 2.3 of Dembo, Eisenstat, and Steihaug [6].

Theorem 2.1. Suppose that Assumption 1 is satisfied. There exists $\varepsilon>0$ such that, if $\left|x_{0}-x_{*}\right| \leq \varepsilon$, the sequence generated by Algorithm 2.1 converges to $x_{*}$ and

$$
\left|x_{k+1}-x_{*}\right|_{*} \leq t\left|x_{k}-x_{*}\right|_{*}
$$


for all $k=0,1,2, \ldots$, where

$$
\left|z_{*}\right|=\left|J\left(x_{*}\right) z\right| .
$$

Proof. See [6]. Observe that, in fact, the hypothesis (2.6) can be weakened.

The next two theorems are the main results of this section. We will assume that the $B_{k}$ 's satisfy a Dennis-Moré condition (see [9]). Under this hypothesis we will prove that the convergence of Algorithm 2.1 is superlinear. If $\left|B_{k}\right|$ and $\left|B_{k}^{-1}\right|$ are bounded, we prove that, eventually, all the iterations satisfy the test (2.2). This means that the number of iterations used by the linear method at Step 3 will be bounded, since the increment (2.1) given by the preconditioner will be accepted for large enough $k$.

Theorem 2.2. Suppose that $F$ satisfies Assumption 1, the sequence $\left(x_{k}\right)$ generated by Algorithm 2.1 converges to $x_{*}$ and

$$
\lim _{k \rightarrow \infty} \frac{\left|\left[B_{k}-J\left(x_{*}\right)\right] s_{k}\right|}{\left|s_{k}\right|}=0
$$

Then

$$
\lim _{k \rightarrow \infty} \frac{\left|x_{k+1}-x_{*}\right|}{\left|x_{k}-x_{*}\right|}=0
$$

Proof. We consider two possibilities:

(i) There exists $k_{0} \in \mathbb{N}$ such that for all $k \geq k_{0}$, the increment $s_{k}$ is computed by (2.4) in Algorithm 2.1.

(ii) For all $k_{0} \in \mathbb{N}$, there exists $k>k_{0}$ such that $s_{k}=s_{k}^{Q}$.

If (i) holds, the algorithm satisfies the conditions of Dembo, Eisenstat, and Steihaug for superlinear convergence of the inexact-Newton method, so (2.12) is proved.

Assume then that (ii) is true. Let $K_{1}$ be the set of indices $k$ such that $s_{k}=s_{k}^{Q}$. Rephrasing the proof of Theorem 2.2 of Dennis and Moré [9], we obtain that

$$
\lim _{k \in K_{1}} \frac{\left|x_{k+1}-x_{*}\right|}{\left|x_{k}-x_{*}\right|}=0
$$

To prove that

$$
\lim _{k \notin K_{1}} \frac{\left|x_{k+1}-x_{*}\right|}{\left|x_{k}-x_{*}\right|}=0
$$

we repeat the arguments in the proof of Theorem 3.3 of [6]. The assertion (2.12) follows from (2.13) and (2.14).

Theorem 2.3. Suppose that $F$ satisfies Assumption 1 and that the sequence $\left(x_{k}\right)$ generated by Algorithm 2.1 converges to $x_{*}$. Assume that the Dennis-Moré condition (2.11) is satisfied and that there exists $M>0$ such that

$$
\left|B_{k}\right| \leq M, \quad\left|B_{k}^{-1}\right| \leq M
$$

for all $k=0,1,2, \ldots$. Then there exists $k_{0} \geq 0$ such that $s_{k}=s_{k}^{Q}$ for all $k \geq k_{0}$, and the convergence is superlinear.

Proof. The superlinear convergence of $\left(x_{k}\right)$ was proved in Theorem 2.2. By (2.6) and the nonsingularity of $J\left(x_{*}\right)$, there exist $k_{0} \in \mathbb{N}, \alpha, \beta>0$ such that, 
for all $k \geq k_{0}$,

$$
\left|F\left(x_{k+1}\right)\right| \leq \beta\left|x_{k+1}-x_{*}\right|
$$

and

$$
\left|F\left(x_{k}\right)\right| \geq \alpha\left|x_{k}-x_{*}\right| \text {. }
$$

(See, for example, [12, Lemma 4.1.15].) So, by (2.12), (2.16), and (2.17),

$$
\lim _{k \rightarrow \infty} \frac{\left|F\left(x_{k+1}\right)\right|}{\left|F\left(x_{k}\right)\right|} \leq \lim _{k \rightarrow \infty} \frac{\beta\left|x_{k+1}-x_{*}\right|}{\alpha\left|x_{k}-x_{*}\right|}=0 .
$$

By the superlinear convergence of $\left(x_{k}\right)$ we have, for large enough $k$,

$$
\left|s_{k}\right| \leq\left|x_{k+1}-x_{*}\right|+\left|x_{k}-x_{*}\right| \leq 2\left|x_{k}-x_{*}\right| \leq \frac{2}{\alpha}\left|F\left(x_{k}\right)\right| .
$$

Then, by (2.19) and (2.11),

$$
\lim _{k \rightarrow \infty} \frac{\left|\left[B_{k}-J\left(x_{*}\right)\right] s_{k}\right|}{\left|F\left(x_{k}\right)\right|} \leq \lim _{k \rightarrow \infty} 2 \frac{\left|\left[B_{k}-J\left(x_{*}\right)\right] s_{k}\right|}{\alpha\left|s_{k}\right|}=0 .
$$

Moreover, by (2.20), (2.15), (2.17), and (2.18),

$$
\begin{aligned}
\lim _{k \rightarrow \infty} & \frac{\left|\left[B_{k}-J\left(x_{*}\right)\right]\left(x_{k}-x_{*}\right)\right|}{\left|F\left(x_{k}\right)\right|} \\
& \leq \lim _{k \rightarrow \infty} \frac{\left|\left[B_{k}-J\left(x_{*}\right)\right] s_{k}\right|}{\left|F\left(x_{k}\right)\right|}+\lim _{k \rightarrow \infty} \frac{\left|\left[B_{k}-J\left(x_{*}\right)\right]\left(x_{k+1}-x_{*}\right)\right|}{\left|F\left(x_{k}\right)\right|} \\
& \leq \lim _{k \rightarrow \infty}\left(\left|B_{k}\right|+\left|J\left(x_{*}\right)\right|\right) \frac{\left|x_{k+1}-x_{*}\right|}{\left|F\left(x_{k}\right)\right|} \\
& \leq \lim _{k \rightarrow \infty} \frac{\left(M+\left|J\left(x_{*}\right)\right|\right)}{\alpha} \frac{\left|F\left(x_{k+1}\right)\right|}{\left|F\left(x_{k}\right)\right|}=0 .
\end{aligned}
$$

So, by (2.21), (2.7), (2.15), (2.17), and (2.12),

$$
\begin{aligned}
\lim _{k \rightarrow \infty} & \frac{\left|\left[B_{k}^{-1}-J\left(x_{*}\right)^{-1}\right]\left[F\left(x_{k+1}\right)-F\left(x_{k}\right)\right]\right|}{\left|F\left(x_{k}\right)\right|} \\
\leq & \lim _{k \rightarrow \infty} \frac{\left.\mid\left[B_{k}^{-1}-J\left(x_{*}\right)^{-1}\right] J\left(x_{*}\right)\left(x_{k+1}\right)-x_{k}\right) \mid}{\left|F\left(x_{k}\right)\right|} \\
& +\lim _{k \rightarrow \infty} \frac{\left|\left[B_{k}^{-1}-J\left(x_{*}\right)^{-1}\right]\left[F\left(x_{k+1}\right)-F\left(x_{k}\right)-J\left(x_{*}\right)\left(x_{k+1}-x_{k}\right)\right]\right|}{\left|F\left(x_{k}\right)\right|} \\
\leq & \lim _{k \rightarrow \infty} \frac{\left|B_{k}^{-1}\left[J\left(x_{*}\right)-B_{k}\right]\left(x_{k+1}-x_{k}\right)\right|}{\left|F\left(x_{k}\right)\right|} \\
& +\frac{\left[M+\left|J\left(x_{*}\right)^{-1}\right|\right] L\left|x_{k+1}-x_{k}\right|\left|x_{k}-x_{*}\right|}{\left|F\left(x_{k}\right)\right|} \\
& \leq \lim _{k \rightarrow \infty} M \frac{\left|\left[B_{k}-J\left(x_{*}\right)\right] s_{k}\right|}{\left|F\left(x_{k}\right)\right|}+\frac{\left[M+\left|J\left(x_{*}\right)^{-1}\right|\right] L\left|x_{k+1}-x_{k}\right|}{\alpha}=0 .
\end{aligned}
$$


Therefore, by (2.15), (2.18), (2.21), and (2.22),

$$
\begin{aligned}
\lim _{k \rightarrow \infty} & \frac{\left|\left[B_{k}^{-1}-J\left(x_{*}\right)^{-1}\right] F\left(x_{k}\right)\right|}{\left|F\left(x_{k}\right)\right|} \\
\leq & \lim _{k \rightarrow \infty} \frac{\left|\left[B_{k}^{-1}-J\left(x_{*}\right)^{-1}\right]\left[F\left(x_{k+1}\right)-F\left(x_{k}\right)\right]\right|}{\left|F\left(x_{k}\right)\right|} \\
& +\lim _{k \rightarrow \infty}\left[M+\left|J\left(x_{*}\right)^{-1}\right|\right] \frac{\left|F\left(x_{k+1}\right)\right|}{\left|F\left(x_{k}\right)\right|}=0 .
\end{aligned}
$$

Hence, by the continuity of $J(x)^{-1}$ in a neighborhood of $x_{*}$,

$$
\lim _{k \rightarrow \infty} \frac{\left|\left[B_{k}^{-1}-J\left(x_{k}\right)^{-1}\right] F\left(x_{k}\right)\right|}{\left|F\left(x_{k}\right)\right|}=0 .
$$

So, by (2.1), (2.24) and the continuity of $J(x)$,

$$
\begin{aligned}
\lim _{k \rightarrow \infty} \frac{\left|J\left(x_{k}\right) s_{k}^{Q}+F\left(x_{k}\right)\right|}{\left|F\left(x_{k}\right)\right|} & =\lim _{k \rightarrow \infty} \frac{\left|J\left(x_{k}\right)\left(-B_{k}^{-1} F\left(x_{k}\right)\right)+F\left(x_{k}\right)\right|}{\left|F\left(x_{k}\right)\right|} \\
& \leq \lim _{k \rightarrow \infty} \frac{\left|J\left(x_{k}\right)\right|\left|\left[B_{k}^{-1}-J\left(x_{k}\right)^{-1}\right] F\left(x_{k}\right)\right|}{\left|F\left(x_{k}\right)\right|}=0 .
\end{aligned}
$$

By (2.25), there exists $k_{0} \in \mathbb{N}$ such that for all $k \geq k_{0}$,

$$
\frac{\left|J\left(x_{k}\right) s_{k}^{Q}+F\left(x_{k}\right)\right|}{\left|F\left(x_{k}\right)\right|} \leq \theta .
$$

So, the test (2.2) is satisfied for all $k \geq k_{0}$. Therefore, for all $k \geq k_{0}$, $s_{k}=s_{k}^{Q}$. This completes the proof.

\section{Structured Least-Change Secant preconditioners}

In $\S 2$ we showed that if $\left|B_{k}\right|$ and $\left|B_{k}^{-1}\right|$ are bounded and $B_{k}$ satisfies the Dennis-Moré condition (2.11), Algorithm 2.1 is locally and superlinearly convergent and, eventually, all the iterations are given by

$$
x_{k+1}=x_{k}-B_{k}^{-1} F\left(x_{k}\right) \text {. }
$$

This means that, using the preconditioner $B_{k}$, the computer work of an inexact-Newton iteration is bounded and superlinear convergence is maintained. Moreover, the results above were obtained without the requirement that the initial $B_{0}$ must be close to the Jacobian.

In this section we will see that preconditioners that satisfy the required properties may be obtained using Martinez's approach. A consequence is that the least-change secant update methods studied by Dennis and Walker may also be used for that purpose (see [27]).

Let $X$ be a finite-dimensional linear space, $F: \Omega \rightarrow \mathbb{R}^{n}, \Omega$ an open and convex set. For all $x, z \in \Omega$ let $\langle,\rangle_{x z}$ be a scalar product over $X$, associated with the norm $\|\cdot\|_{x z}$. Let $D \subset \Omega \times X$ be an open set and $\varphi: D \rightarrow \mathbb{R}^{n \times n}$ a continuous function. For all $x, z \in \Omega$, let $V(x, z) \subset X$ be an affine subspace. The following algorithm describes an inexact-Newton method preconditioned by a structured Least-Change Secant procedure. 
Algorithm 3.1. Let $x_{0} \in \Omega$ be a given initial approximation, $E_{0} \in X, \theta_{k} \in$ $(0, \theta)$ for all $k=0,1,2, \ldots, 0<\theta<t<1$ and $\lim _{k \rightarrow \infty} \theta_{k}=0$. The steps for obtaining $x_{k+1}, E_{k+1}, k=0,1,2, \ldots$, are

Step 1. If $\left(x_{k}, E_{k}\right) \in D$ and $B_{k}$ is nonsingular, where

$$
B_{k}=\varphi\left(x_{k}, E_{k}\right) \text {, }
$$

define

$$
s_{k}^{Q}=-B_{k}^{-1} F\left(x_{k}\right)
$$

Else, go to Step 3.

If

$$
\left|J\left(x_{k}\right) s_{k}^{Q}+F\left(x_{k}\right)\right| \leq \theta\left|F\left(x_{k}\right)\right|,
$$

define

$$
s_{k}=s_{k}^{Q}
$$

and go to Step 4.

Step 3. Find an increment $s_{k}$ such that

$$
\left|J\left(x_{k}\right) s_{k}+F\left(x_{k}\right)\right| \leq \theta_{k}\left|F\left(x_{k}\right)\right|,
$$

using some iterative method.

Step 4. Define

$$
x_{k+1}=x_{k}+s_{k}
$$

Step 5. Compute

$$
E_{k+1}=P_{k}\left(E_{k}\right),
$$

where $P_{k}$ is the orthogonal projector on $V\left(x_{k}, x_{k+1}\right)$ with respect to $\|\cdot\|_{x_{k} x_{k+1}}$.

Clearly, the local linear convergence Theorem 2.1 holds for Algorithm 3.1, if $F$ satisfies Assumption 1. Superlinear convergence will be a consequence of the following assumptions.

Assumption 2. Let $\|\cdot\|$ be a fixed norm on $X$ associated with the scalar product $\langle$,$\rangle , let E_{*} \in X$ and let $c_{1} \geq 0$ be a constant. We assume that for all $x, z \in \Omega$, there exists $E=E(x, z) \in V(x, z)$ such that

$$
\left\|E-E_{*}\right\| \leq c_{1} \sigma(x, z),
$$

where $\sigma(x, z)$ is defined by $(2.8)$.

Assumption 3. There exists $c_{2} \geq 0$ such that, for all $x, z \in \Omega, E \in X$,

$$
\|E\|_{x z} \leq\left[1+c_{2} \sigma(x, z)\right]\|E\|
$$

and

$$
\|E\| \leq\left[1+c_{2} \sigma(x, z)\right]\|E\|_{x z} .
$$

Remark. In Martinez's paper [26] it is assumed that $\left(x_{*}, E_{*}\right)$ belongs to the domain of $\varphi$ and that $\left|I-\varphi\left(x_{*}, E_{*}\right)^{-1} J\left(x_{*}\right)\right| \leq r_{*}<1$. These assumptions will not be necessary to prove the main results of the present work. 
Lemma 3.1. Let $F$ satisfy Assumption 1 , and let $V, E_{*},\|\cdot\|_{x z}$, and $\|\cdot\|$ satisfy Assumptions 2 and 3. Suppose that the sequence $\left(x_{k}\right)$ generated by Algorithm 3.1 converges to $x_{*}$ and satisfies

$$
\left|x_{k+1}-x_{*}\right|_{*} \leq t\left|x_{k}-x_{*}\right|_{*}
$$

for all $k \geq k_{0}$. Then, $\left\|E_{k}\right\|$ is bounded.

Proof. By (3.10), we have that

$$
\left\|E_{k+1}-E_{*}\right\| \leq\left[1+c_{2} \sigma\left(x_{k}, x_{k+1}\right)\right]\left\|E_{k+1}-E_{*}\right\|_{k}
$$

for all $k=0,1,2, \ldots$, where $\|\cdot\|_{k} \equiv\|\cdot\|_{x_{k} x_{k+1}}$.

Now, by (3.12) and the equivalence of norms on $\mathbb{R}^{n}$, there exists $c_{*}>0$ such that

$$
\sigma\left(x_{k}, x_{k+1}\right) \leq c_{*}\left|x_{k}-x_{*}\right|
$$

for all $k \geq k_{0}$. Thus, by (3.13) and (3.14),

$$
\left\|E_{k+1}-E_{*}\right\| \leq\left(1+c_{2}^{\prime}\left|x_{k}-x_{*}\right|_{*}\right)\left\|E_{k+1}-E_{*}\right\|_{k}
$$

for all $k \geq k_{0}$, where $c_{2}^{\prime}=c_{2} c_{*}$.

Let $\widehat{E}_{k}$ be the orthogonal projection of $E_{*}$ on $V\left(x_{k}, x_{k+1}\right)$, related to the norm $\|\cdot\|$. By (3.15),

$$
\left\|E_{k+1}-E_{*}\right\| \leq\left(1+c_{2}^{\prime}\left|x_{k}-x_{*}\right|_{*}\right)\left[\left\|E_{k+1}-\widehat{E}_{k}\right\|_{k}+\left\|\widehat{E}_{k}-E_{*}\right\|_{k}\right]
$$

for all $k \geq k_{0}$. But $E_{k+1}$ is the projection of $E_{k}$ on $V\left(x_{k}, x_{k+1}\right)$, and $\widehat{E}_{k} \in$ $V\left(x_{k}, x_{k+1}\right)$. So,

$$
\left\|E_{k+1}-\widehat{E}_{k}\right\|_{k} \leq\left\|E_{k}-\widehat{E}_{k}\right\|_{k} \leq\left\|E_{k}-E_{*}\right\|_{k}+\left\|\widehat{E}_{k}-E_{*}\right\|_{k} .
$$

Hence, by (3.10), (3.14), (3.16), and (3.17),

$$
\begin{aligned}
\left\|E_{k+1}-E_{*}\right\| & \leq\left(1+c_{2}^{\prime}\left|x_{k}-x_{*}\right|_{*}\right)\left[\left\|E_{k}-E_{*}\right\|_{k}+2\left\|\widehat{E}_{k}-E_{*}\right\|_{k}\right] \\
& \leq\left(1+c_{2}^{\prime}\left|x_{k}-x_{*}\right|_{*}\right)^{2}\left[\left\|E_{k}-E_{*}\right\|+2\left\|\widehat{E}_{k}-E_{*}\right\|\right]
\end{aligned}
$$

for $k \geq k_{0}$.

Now, by Assumption 2, $\left\|\widehat{E}_{k}-E_{*}\right\| \leq c_{1} \sigma\left(x_{k}, x_{k+1}\right)$. Therefore, by (3.18) and (3.14),

$$
\begin{aligned}
\left\|E_{k+1}-E_{*}\right\| & \leq\left(1+c_{2}^{\prime}\left|x_{k}-x_{*}\right|_{*}\right)^{2}\left[\left\|E_{k}-E_{*}\right\|+2 c_{1} \sigma\left(x_{k}, x_{k+1}\right)\right] \\
& \leq\left(1+c_{2}^{\prime}\left|x_{k}-x_{*}\right|_{*}\right)^{2}\left[\left\|E_{k}-E_{*}\right\|+2 c_{1} c_{*}\left|x_{k}-x_{*}\right|_{*}\right] .
\end{aligned}
$$

Thus, setting $d_{1}=\left|x_{k_{0}}-x_{*}\right|_{*}$, we obtain that there exist $c_{3}, c_{4}>0$ such that

$$
\left\|E_{k+1}-E_{*}\right\| \leq\left(1+c_{3}\left|x_{k}-x_{*}\right|_{*}\right)|| E_{k}-E_{*} \|+c_{4}\left|x_{k}-x_{*}\right|_{*}
$$

for all $k \geq k_{0}$. So, by (3.12),

$$
\left\|E_{k+1}-E_{*}\right\| \leq\left(1+c_{3} t^{k-k_{0}} d_{1}\right)\left\|E_{k}-E_{*}\right\|+c_{4} t^{k-k_{0}} d_{1}
$$

for all $k \geq k_{0}$. So, by Lemma 3.3 of [9], $\left\|E_{k}-E_{*}\right\|$ is bounded, and hence $\left\|E_{k}\right\|$ is bounded.

Lemma 3.2. Assume the hypotheses of Lemma 3.1. There exists $c_{5}>0$ such that

$$
\left\|E_{k+j}-E_{*}\right\| \leq\left\|E_{k}-E_{*}\right\|+c_{5}\left|x_{k}-x_{*}\right|_{*}
$$

for all $k \geq k_{0}, j=1,2,3, \ldots$. 
Proof. By Lemma 3.1, there exists $d_{2}>0$ such that

$$
\left\|E_{k}-E_{*}\right\| \leq d_{2}
$$

for all $k=0,1,2, \ldots$ Thus, by (3.20) and (3.23),

$$
\begin{aligned}
\left\|E_{k+1}-E_{*}\right\| & \leq\left(1+c_{3}\left|x_{k}-x_{*}\right|_{*}\right)\left\|E_{k}-E_{*}\right\|+c_{4}\left|x_{k}-x_{*}\right|_{*} \\
& \leq\left\|E_{k}-E_{*}\right\|+\left(c_{3} d_{2}+c_{4}\right)\left|x_{k}-x_{*}\right|_{*} \\
& =\left\|E_{k}-E_{*}\right\|+c_{6}\left|x_{k}-x_{*}\right|_{*}
\end{aligned}
$$

for all $k \geq k_{0}$, where $c_{6}=c_{3} d_{2}+c_{4}$. So, by (3.12) and (3.24),

$$
\begin{aligned}
\left\|E_{k+j}-E_{*}\right\| & \leq\left\|E_{k}-E_{*}\right\|+\sum_{l=0}^{j-1} c_{6}\left|x_{k+l}-x_{*}\right|_{*} \\
& \leq\left\|E_{k}-E_{*}\right\|+\sum_{l=0}^{j-1} c_{6} t^{l}\left|x_{k}-x_{*}\right|_{*} \\
& =\left\|E_{k}-E_{*}\right\|+c_{6}\left|x_{k}-x_{*}\right|_{*}^{j-1} \sum_{l=0}^{l} \\
& \leq\left\|E_{k}-E_{*}\right\|+c_{5}\left|x_{k}-x_{*}\right|_{*}
\end{aligned}
$$

for all $k \geq k_{0}, j=0,1,2, \ldots$, where $c_{5}=c_{6} /(1-t)$.

Lemma 3.3. Assume the hypotheses of Lemma 3.1. There exists $c_{7}>0$ such that for all $k \geq k_{0}, j=1,2,3, \ldots$,

$$
\left\|E_{k+j}-E_{*}\right\|^{2} \leq\left\|E_{k}-E_{*}\right\|^{2}+c_{7}\left|x_{k}-x_{*}\right|_{*} .
$$

Proof. Trivial, using (3.22) and the boundedness of $\left\|E_{k}-E_{*}\right\|$.

Theorem 3.1. Assume the hypotheses of Lemma 3.1. Then

$$
\lim _{k \rightarrow \infty}\left\|E_{k+1}-E_{k}\right\|=0 .
$$

Proof. This proof reproduces the arguments of the proof of Theorem 3.3 of [26], using (3.26) for proving the formula (3.32) of Martínez's paper.

Theorem 3.2. Assume the hypotheses of Lemma 3.1. Suppose that there exists a closed set $G \subset \mathbb{R}^{n} \times X$ such that $\left(x_{k}, E_{k}\right) \in G \subset D$ for all $k=0,1,2, \ldots$. Then

$$
\lim _{k \rightarrow \infty}\left|\varphi\left(x_{k+1}, E_{k+1}\right)-\varphi\left(x_{k}, E_{k}\right)\right|=0 .
$$

Proof. Since $\left\|E_{k}\right\|$ is uniformly bounded and $\left(x_{k}\right)$ is convergent, there exists a compact set $G^{\prime}$ such that $\left(x_{k}, E_{k}\right) \in G^{\prime}$ for all $k=0,1,2, \ldots$. So, $G \cap G^{\prime} \subset$ $D$ is compact and $\varphi$ is uniformly continuous in $G \cap G^{\prime}$. Therefore, (3.28) follows from (3.27) and from $\lim _{k \rightarrow \infty}\left|x_{k+1}-x_{k}\right|=0$.

Theorem 3.3. Let $F$ satisfy Assumption 1 and let $V, E_{*},\|\cdot\|_{x z}$, and $\|\cdot\|$ satisfy Assumptions 2 and 3. Suppose that the sequence $\left(x_{k}\right)$ generated by Algorithm 3.1 converges to $x_{*}$ and satisfies (3.12). Assume that there exists a closed set 
$G \subset \mathbb{R}^{n} \times X$ such that $\left(x_{k}, E_{k}\right) \in G \subset D$ for all $k=0,1,2, \ldots, B_{k}$ is nonsingular for all $k=0,1,2, \ldots$ and $\left|B_{k}^{-1}\right|$ is bounded. Suppose that

$$
\lim _{k \rightarrow \infty} \frac{\left|\left[B_{k+1}-J\left(x_{*}\right)\right] s_{k}\right|}{\left|s_{k}\right|}=0 .
$$

Then there exists $k_{1} \in \mathbb{N}$ such that $s_{k}=s_{k}^{Q}$ for all $k \geq k_{1}$, and convergence is superlinear.

Proof. We prove that the hypotheses of Theorem 2.3 are satisfied.

By (3.29) and (3.28),

$$
\lim _{k \rightarrow \infty} \frac{\left|\left[B_{k}-J\left(x_{*}\right)\right] s_{k}\right|}{\left|s_{k}\right|} \leq \lim _{k \rightarrow \infty}\left|B_{k+1}-B_{k}\right|+\lim _{k \rightarrow \infty} \frac{\left|\left[B_{k+1}-J\left(x_{*}\right)\right] s_{k}\right|}{\left|s_{k}\right|}=0 .
$$

So, the Dennis-More condition (2.11) holds here. Now, by Lemma 3.2, there exists $M>0$ such that $\left\|E_{k}\right\| \leq M$ for all $k=0,1,2, \ldots$. Then, for all $k=0,1,2, \ldots$,

$$
E_{k} \in C_{2} \equiv\{E \in G \mid\|E\| \leq M\} .
$$

Now, since $\left(x_{k}\right)$ is convergent, the set $\left\{x_{0}, x_{1}, x_{2}, \ldots\right\}$ is contained in a compact set $C_{1}$. Since $C_{1} \times C_{2}$ is compact and $\varphi$ is continuous, $\varphi(x, E)$ is bounded for $(x, E) \in\left(C_{1} \times C_{2}\right) \cap G$. Therefore, $\left|B_{k}\right|$ is bounded. Since $\left|B_{k}^{-1}\right|$ is bounded by hypothesis, the desired result follows from Theorem 2.3.

Theorem 3.4. Assume the hypotheses of Theorem 3.3 except that instead of (3.29) we assume that

$$
B_{k+1} s_{k}=y_{k} \equiv F\left(x_{k+1}\right)-F\left(x_{k}\right)
$$

for all $k=0,1,2, \ldots$. Then there exists $k_{1} \in \mathbb{N}$ such that $s_{k}=s_{k}^{Q}$ for all $k \geq k_{1}$, and convergence is superlinear.

Proof. By (2.7) the secant equation (3.30) implies (3.29). So, the desired result follows from Theorem 3.3.

\section{AUGMENTED BFGS PRECONDITIONERS}

In this section we consider nonlinear systems $F(x)=0$ where the Jacobian matrix $J(x)$ is symmetric and positive definite. Typical examples of this type of system come from minimization problems. Since $J\left(x_{k}\right)$ is symmetric and positive definite, incomplete Cholesky factorizations are natural preconditioners of the system (1.2). However, since we need to solve a sequence of systems of type (1.2), it is natural to modify the incomplete Cholesky preconditioner by some least-change secant formula. In this section we analyze the modification of a generic preconditioner by a BFGS-type formula. Other modifications may also be considered, such as PSB modifications or DFP structured modifications (see $[12,14,7,41])$.

Assume, as always, that $F \in C^{1}(\Omega)$, where $\Omega$ is an open and convex set. Let $C: \Omega \rightarrow \mathbb{R}^{n \times n}$ be a continuous function. Assume that linear systems whose matrix is $C(x)$ are easy to solve. Assume that $C(x)=\mathscr{L}(x) \mathscr{L}(x)^{\top}$, where $\mathscr{L}(x)$ is lower triangular and has a simple structure. The product $\mathscr{L}(x) \mathscr{L}(x)^{\top}$ may be considered an approximation of the Cholesky factorization of $J(x)$. A 
particular case is when $C(x)$ is the matrix that defines some stationary linear iterative method, such as Jacobi or SOR (see [28, 22, etc.]). For example, the preconditioner $C(x)$ induced by the Jacobi method is the diagonal of $J(x)$.

Below we define the main algorithm of this section.

Algorithm 4.1. Let $x_{0} \in \Omega$ be a given initial approximation to the solution of (1.1) such that $C\left(x_{0}\right)$ is nonsingular, $E_{0} \in \mathbb{R}^{n \times n}, 0<\theta<t<1, \theta_{k} \in$ $(0, \theta)$ for all $k=0,1,2, \ldots$ and $\lim _{k \rightarrow \infty} \theta_{k}=0$. Given $x_{k}, E_{k}$ such that $x_{k} \in \Omega$ and $C\left(x_{k}\right)$ is nonsingular, the steps for obtaining $x_{k+1}, E_{k+1}$, $k=0,1,2, \ldots$, are:

Step 1. Define

$$
s_{k}^{Q}=-\left[C\left(x_{k}\right)^{-1}+E_{k}\right] F\left(x_{k}\right) .
$$

Step 2. If

$$
\left|J\left(x_{k}\right) s_{k}^{Q}+F\left(x_{k}\right)\right| \leq \theta\left|F\left(x_{k}\right)\right|,
$$

define

and go to Step 4.

$$
s_{k}=s_{k}^{Q}
$$

Step 3. Find an increment $s_{k}$ such that

$$
\left|J\left(x_{k}\right) s_{k}+F\left(x_{k}\right)\right| \leq \theta_{k}\left|F\left(x_{k}\right)\right| .
$$

Step 4. Define

$$
x_{k+1}=x_{k}+s_{k}
$$

Step 5. Define

$$
\begin{aligned}
y_{k} & =F\left(x_{k+1}\right)-F\left(x_{k}\right), \\
s_{k}^{\#} & =s_{k}-C\left(x_{k+1}\right)^{-1} y_{k} .
\end{aligned}
$$

If $s_{k}^{\top} y_{k} \leq 0$, set $E_{k+1}=E_{k}$, else compute

$$
E_{k+1}=E_{k}+\frac{\left(s_{k}^{\#}-E_{k} y_{k}\right) s_{k}^{\top}+s_{k}\left(s_{k}^{\#}-E_{k} y_{k}\right)^{\top}}{s_{k}^{\top} y_{k}}-\frac{\left(s_{k}^{\#}-E_{k} y_{k}\right)^{\top} y_{k} s_{k} s_{k}^{\top}}{\left(s_{k}^{\top} y_{k}\right)^{2}}
$$

Remarks. At Step 3, the increment $s_{k}$ is calculated in an unspecified manner. However, the natural way to compute it is to use a sufficient number of steps of the Conjugate Gradient method, preconditioned by $C\left(x_{k}\right)^{-1}+E_{k}$. In fact, following Algorithm 10.3.1 of [19], we observe that $s_{k}^{Q}=\left(C\left(x_{k}\right)^{-1}+E_{k}\right) F\left(x_{k}\right)$ must be necessarily computed at the first step of the preconditioned $C G$ algorithm if $M=\left[C\left(x_{k}\right)^{-1}+E_{k}\right]^{-1}$. Therefore, the work done at Step 1 of Algorithm 4.1 is incorporated naturally in the preconditioned $C G$ procedure.

The structured BFGS formula (4.7) is derived by requiring $E_{k+1}$ to be the closest symmetric matrix to $E_{k}$ that satisfies the secant equation

$$
\left(C\left(x_{k+1}\right)^{-1}+E_{k+1}\right) y_{k}=s_{k},
$$

in the weighted Frobenius norm defined by (4.13) below. For details, see, for example, $[12,14,26]$.

Using the results of $\S \S 2$ and 3, we prove the following convergence results for Algorithm 4.1. 
Theorem 4.1. Suppose that Assumption 1 is satisfied. There exists $\varepsilon>0$ such that, if $\left|x_{0}-x_{*}\right| \leq \varepsilon$, the sequence generated by Algorithm 4.1 converges to $x_{*}$. Moreover,

$$
\left|x_{k+1}-x_{*}\right|_{*} \leq t\left|x_{k}-x_{*}\right|_{*}
$$

for all $k=0,1,2, \ldots$, where $|z|_{*}=\left|J\left(x_{*}\right) z\right|$.

Proof. This result is an application of Theorem 3.3 of [6].

Lemma 4.1. Let $F$ satisfy Assumption 1 and let $J\left(x_{*}\right)$ be symmetric and positive definite. Suppose that the sequence generated by Algorithm 4.1 converges to $x_{*}$ and that $x_{k} \neq x_{*}$ for all $k=0,1,2, \ldots$. There exists $k_{0} \in \mathbb{N}$ such that $s_{k}^{\top} y_{k}>0$ for all $k \geq k_{0}$. So, for all $k \geq k_{0}$, the matrix $E_{k+1}$ is computed using (4.7).

Proof. Define

$$
\lambda=\min _{\substack{\omega \in \mathbb{R}^{n} \\ \omega \neq 0}} \frac{\omega^{\top} J\left(x_{*}\right) \omega}{\omega^{\top} \omega}>0 .
$$

Since $s_{k} \neq 0$ for all $k=0,1,2, \ldots$, we have, by (2.7), that

$$
\begin{aligned}
\frac{s_{k}^{\top} y_{k}}{s_{k}^{\top} s_{k}} & =\frac{s_{k}^{\top}\left[F\left(x_{k+1}\right)-F\left(x_{k}\right)\right]}{s_{k}^{\top} s_{k}} \\
& =\frac{s_{k}^{\top}\left[F\left(x_{k+1}\right)-F\left(x_{k}\right)-J\left(x_{*}\right) s_{k}\right]}{s_{k}^{\top} s_{k}}+\frac{s_{k}^{\top} J\left(x_{*}\right) s_{k}}{s_{k}^{\top} s_{k}} \\
& \geq-\frac{L\left|s_{k}\right|^{2} \sigma\left(x_{k}, x_{k+1}\right)}{s_{k}^{\top} s_{k}}+\frac{s_{k}^{\top} J\left(x_{*}\right) s_{k}}{s_{k}^{\top} s_{k}} \geq \lambda-L \sigma\left(x_{k}, x_{k+1}\right) .
\end{aligned}
$$

So, for large enough $k$, we have $s_{k}^{\top} y_{k}>0$, as we wanted to prove.

Theorem 4.2. Let $F$ satisfy Assumption 1, and let $C\left(x_{*}\right)$ be nonsingular. Suppose that the sequence generated by Algorithm 4.1 converges to $x_{*}$ and satisfies (4.8). There exist $k_{0} \in \mathbb{N}$ and $\Delta>0$ such that, if

$$
\left|E_{k_{1}}-E_{*}\right| \leq \Delta
$$

for some $k_{1} \geq k_{0}$, where

$$
E_{*}=J\left(x_{*}\right)^{-1}-C\left(x_{*}\right)^{-1},
$$

then convergence is superlinear and $s_{k}=s_{k}^{Q}$ for all $k$ large enough.

Proof. Define $X=\mathbb{R}^{n \times n}$. For $x, z \in \Omega$ we define

$$
H(x, z)=\int_{0}^{1} J(x+t(z-x)) d t .
$$

Possibly restricting $\Omega$, we can assume, since $J\left(x_{*}\right)$ is positive definite, that $H(x, z)$ is positive definite for all $x, z \in \Omega$. Define

$$
\|E\|_{x z}=\left\|L(x, z)^{\top} E L(x, z)\right\|_{F},
$$

where $L(x, z) L(x, z)^{\top}$ is the Cholesky factorization of $H(x, z)$, and

$$
\|E\|=\left\|L_{*}^{\top} E L_{*}\right\|_{F},
$$


where $L_{*} L_{*}^{\top}$ is the Cholesky factorization of $J\left(x_{*}\right)$. Assumption 3 can be seen to hold, using the arguments in the proof of Theorem 4.3 of [26].

Define

$$
V(x, z)=S \cap\left\{E \in X \mid E[F(z)-F(x)]=z-x-C(z)^{-1}[F(z)-F(x)]\right\}
$$

where $S$ is the subspace of symmetric matrices of $\mathbb{R}^{n \times n}$. By Lemma 4.1 we can assume, without loss of generality, that $s_{k}^{\top} y_{k}>0$ for all $k \geq 0$. Thus, repeating the arguments of [26, pp. 150-151], we see that

$$
E_{k+1}=P_{k}\left(E_{k}\right)
$$

for all $k=0,1,2, \ldots$, where $P_{k}$ is the projection operator on $V\left(x_{k}, x_{k+1}\right)$ with respect to $\|\cdot\|_{x_{k} x_{k+1}}$.

Finally, define

$$
E(x, z)=\left[\int_{0}^{1} J(x+t(z-x)) d t\right]^{-1}-C(z)^{-1} .
$$

Obviously, $E(x, z) \in V(x, z)$, and (3.9) follows from (2.6) and Banach's lemma ([19, p. 59]). Therefore, Assumptions 2 and 3 hold for Algorithm 4.1.

Define

$$
\varphi(x, E)=\left(C(x)^{-1}+E\right)^{-1}
$$

and

$$
D=\left\{(x, E) \in \Omega \times X \mid C(x) \text { and } C(x)^{-1}+E \text { are nonsingular }\right\} .
$$

Clearly, $\left(x_{*}, E_{*}\right) \in D$ in this case.

Define $c_{5}$ as in Lemma 3.2. By (3.21), we have

$$
\left\|E_{k+j}-E_{*}\right\| \leq\left\|E_{k}-E_{*}\right\|+c_{5}\left|x_{k}-x_{*}\right|_{*}
$$

for all $k \geq 0, j=0,1,2, \ldots$.

Let $\varepsilon_{3}, \Delta_{1}>0$ be such that

$$
G \equiv\left\{(x, E) \in \Omega \times X|| x-\left.x_{*}\right|_{*} \leq \varepsilon_{3},\left\|E-E_{*}\right\| \leq \Delta_{1}\right\} \subset D .
$$

Let $k_{0} \in \mathbb{N}, \Delta>0$ be such that

$$
\Delta+c_{5}\left|x_{k_{0}}-x_{*}\right|_{*} \leq \Delta_{1}
$$

and

$$
\left|x_{k_{0}}-x_{*}\right|_{*} \leq \varepsilon_{3} .
$$

By (4.8), (4.10) and (4.20)-(4.23), $\left(x_{k}, E_{k}\right)$ belongs to the compact set $G$ for all $k \geq k_{1}$. Clearly, $\left|C(x)^{-1}+E\right|$ is bounded for $(x, E) \in G$, and the desired result follows from Theorem 3.2.

Remark. Observe that the restriction (4.10) on some "initial" $E_{k}$ does not have the same meaning as the constraint $\left\|E_{0}-E_{*}\right\| \leq \delta$ in the hypothesis of Theorem 3.2 of [26]. The latter was a very severe restriction that guarantees that all the $E_{k}$ 's belong to a small neighborhood of $E_{*}$ where all the parameters generate 
contractive mappings. In the case of (4.10) we only want to guarantee that deterioration is not sufficient to produce unbounded $\left|B_{k}\right|$ or $\left|B_{k}^{-1}\right|$.

\section{ConClusions}

The computer time which corresponds to the resolution of a large-scale linear system of equations using a direct method is not negligible. Sometimes, the associated cost largely dominates the cost of computing the function and the derivatives.

For this reason, traditional quasi-Newton methods like the sparse Broyden method $([2,38])$ tend to be rarely used because they need the same linear algebra work as Newton's method. However, in some quasi-Newton methods the computer time used to solve $B_{k} s=-F(x)$ is substantially less than the computer time needed to solve (1.2). These methods are still very useful. Essentially, these algorithms are low-rank modification methods (see [12]) and methods based on direct updating of factorizations ([8, 23, 24, 25, 42, 43, 20]). These "cheap linear algebra" quasi-Newton methods can be used as preconditioners when $C G$-type algorithms are applied to (1.2). Of course, it is not possible to claim that all these algorithms generate good preconditioners in practice for the inexact-Newton method. However, we proved in this paper that the updating schemes that fall under the Martínez theory ([26]) have very nice theoretical properties as inexact-Newton preconditioners. With this contribution, we support the point of view that quasi-Newton and inexact-Newton methods are not competitors, but complement each other for solving large-scale nonlinear systems.

The idea of using secant modifications of classical preconditioners, like the one introduced in $\S 4$, is promising because it tends to make the best possible use of available information at each iteration of the inexact-Newton method. The theory introduced in this paper encourages us to initiate a comprehensive set of experiments with the aim to discover the best updating schemes and to evaluate particular cases of this approach in practical large-scale problems. Computer implementations of Algorithm 2.1 will probably need suitable safeguards in order to keep $\left|B_{k}\right|$ and $\left|B_{k}^{-1}\right|$ bounded, since we cannot predict how far $x_{0}$ is from $x_{*}$. A large initial error can, in theory, produce singular (or nearly singular) preconditioners. Moreover, owing to poor initial estimates, a large number of iterations could be necessary to satisfy (2.2). Only computer experimentation can tell us if these are serious drawbacks.

\section{ACKNOWLEDGMENTS}

The author is indebted to a referee and the associate editor for useful suggestions.

\section{BIBLIOGRAPHY}

1. O. Axelsson, A survey of preconditioned iterative methods for linear systems of algebraic equations, BIT 25 (1985), 166-187.

2. C. G. Broyden, The convergence of an algorithm for solving sparse nonlinear systems, Math. Comp. 25 (1971), 285-294.

3. C. G. Broyden, J. E. Dennis, Jr., and J. J. Moré, On the local and superlinear convergence of quasi-Newton methods, J. Inst. Math. Appl. 12 (1973), 223-245. 
4. A. R. Conn, N. Gould, and Ph. L. Toint, A comprehensive description of LANCELOT, Technical Rep. Hatfield Polytechnique Center, 1990.

5. E. J. Dean, A Model Trust Region Modification of Inexact Newton's method for nonlinear two point boundary value problems, TR No. 85-6, Dept. of Math. Sci., Rice University, Houston, Texas, 1985.

6. R. S. Dembo, S. C. Eisenstat, and T. Steihaug, Inexact Newton methods, SIAM J. Numer. Anal. 14 (1982), 400-408.

7. J. E. Dennis, H. J. Martinez, and R. A. Tapia, A convergence theory for the structured BFGS secant method with an application to nonlinear least squares, Technical Rep. 87-15, Dept. of Math. Sci., Rice University, Houston, Texas, 1988.

8. J. E. Dennis and E. S. Marwil, Direct secant updates of matrix factorizations, Math. Comp. 38 (1982), 459-476.

9. J. E. Dennis and J. J. Moré, A characterization of superlinear convergence and its application to quasi-Newton methods, Math. Comp. 28 (1974), 549-560.

10. __ Quasi-Newton methods, motivation and theory, SIAM Rev. 19 (1977), 46-89.

11. J. E. Dennis and R. B. Schnabel, Least change secant updates for quasi-Newton methods, SIAM Rev. 21 (1979), 443-459.

12. __ Numerical methods for unconstrained optimization and nonlinear equations, PrenticeHall, Englewood Cliffs, NJ, 1983.

13. J. E. Dennis and K. Turner, Generalized conjugate directions, Linear Algebra Appl. 88/89 (1986), 187-209.

14. J. E. Dennis and H. F. Walker, Convergence theorems for least-change secant update methods, SIAM J. Numer. Anal. 18 (1981), 949-987.

15. I. S. Duff, MA28-a set of Fortran subroutines for sparse unsymmetric linear equations, AERE R8730, Her Majesty's Stationery Office, London, 1977.

16. I. S. Duff, A. M. Erisman, and J. K. Reid, Direct methods for sparse matrices, Clarendon Press, Oxford, 1986.

17. A. George and E. Ng, Symbolic factorization for sparse Gaussian elimination with partial pivoting, SIAM J. Sci. Statist. Comput. 8 (1987), 877-898.

18. G. H. Golub and D. P. O'Leary, Some history of the Conjugate Gradient and Lanczos Algorithms: 1948-1976, SIAM Rev. 31 (1989), 50-102.

19. G. H. Golub and Ch. F. Van Loan, Matrix computations, The Johns Hopkins Univ. Press, Baltimore and London, 1989.

20. M. A. Gomes-Ruggiero, J. M. Martínez, and A. C. Moretti, Comparing algorithms for solving sparse nonlinear systems of equations, SIAM J. Sci. Statist. Comput. 13 (1992), 459-483.

21. M. R. Hestenes and E. Stiefel, Methods of conjugate gradients for solving linear systems, J. Res. Nat. Bur. Standards B49 (1952), 409-436.

22. D. S. Kershaw, The incomplete Cholesky-Conjugate Gradient method for the iterative solution of systems of linear equations, J. Comput. Phys. 26 (1978), 43-65.

23. J. M. Martinez, A quasi-Newton method with a new updating for the LDU factorization of the approximate Jacobian, Mat. Apl. Comput. 2 (1983), 131-142.

24. __ Quasi-Newton methods with factorization scaling for solving sparse nonlinear systems of equations, Computing 38 (1987), 133-141.

25. __ A family of quasi-Newton methods for nonlinear equations with direct secant updates of matrix factorizations, SIAM J. Numer. Anal. 27 (1990), 1034-1049.

26. L L L Lal convergence theory of inexact Newton methods based on structured least-change updates, Math. Comp. 55 (1990), 143-168.

27. _ On the relation between two local convergence theories of least-change secant update methods, Math. Comp. 59 (1992), 457-481.

28. J. A. Meijerink and H. A. van der Vorst, An iterative solution method for linear systems of which the coefficient matrix is a symmetric M-matrix, Math. Comp. 3 (1977), 148-162.

29. S. G. Nash, Newton-type minimization via the Lanczos method, SIAM J. Numer. Anal. 21 (1984), 770-787. 
30. __ Preconditioning of truncated-Newton methods, SIAM J. Sci. Statist. Comput. 6 (1985), 599-616.

31. L. Nazareth and J. Nocedal, A study of conjugate-gradient methods, Report SOL 78-29, Dept. of Operations Res., Stanford University, Stanford, CA, 1978.

32. J. M. Ortega and W. C. Rheinboldt, Iterative solution of nonlinear equations in several variables, Academic Press, New York, 1970.

33. A. M. Ostrowski, Solution of equations in Euclidean and Banach spaces, Academic Press, New York, 1973.

34. F. A. Potra and V. Pták, Sharp error bounds for Newton's process, Numer. Math. 34 (1980), 63-72.

35. V. Pták, The rate of convergence of Newton's process, Numer. Math. 25 (1976), 279-285.

36. Y. Saad, Krylov subspace methods of supercomputers, SIAM J. Sci. Statist. Comput. 10 (1989), 1200-1232.

37. Y. Saad and M. H. Schultz, GMRES: a generalized minimal residual algorithm for solving nonsymmetric linear systems, SIAM J. Sci. Statist. Comput. 7 (1986), 856-869.

38. L. K. Schubert, Modification of a quasi-Newton method for nonlinear equations with a sparse Jacobian, Math. Comp. 24 (1970), 27-30.

39. H. Schwetlick, Numerische Lösung nichtlinearer Gleichungen, Deutscher Verlag der Wissenschaften, Berlin, 1978.

40. A. H. Sherman, On Newton-iterative methods for the solution of systems of nonlinear equations, SIAM J. Numer. Anal. 15 (1978), 755-771.

41. R. A. Tapia, On secant updates for use in general constrained optimization, Technical Rep. 84-3, Dept. of Math. Sci., Rice University, Houston, Texas, 1987.

42. R. P. Tewarson, A new quasi-Newton algorithm, Appl. Math. Lett. 1 (1988), 101-104.

43. R. P. Tewarson and Y. Zhang, Sparse quasi-Newton LDU update, Internat. J. Numer. Methods Engrg. 24 (1987), 1093-1100.

44. D. M. Young, A historical overview of iterative methods, Comput. Phys. Comm. 53 (1989), 1-18.

45. Z. Zlatev, J. Wasniewski, and K. Schaumburg, Y12M. Solution of large and sparse systems of linear algebraic equations, Lecture Notes in Comput. Sci., vol. 121, Springer-Verlag, Berlin and New York, 1981.

Department of Applied Mathematics, IMECC-UNiCAMP, State University of CamPINAS, CP 6065, 13081 CAMPINAS SP, BRAZIL

E-mail address: martinez@ccvax.unicamp.ansp.br 